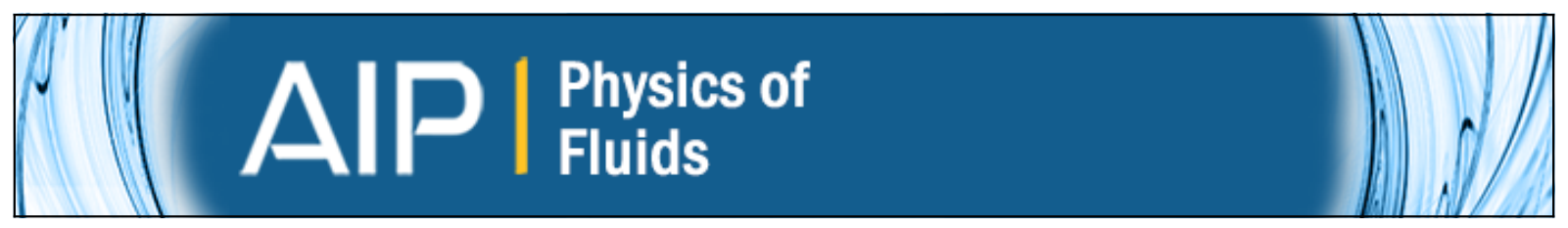

Experiments on wind-perturbed rogue wave hydrodynamics using the Peregrine breather model

A. Chabchoub, N. Hoffmann, H. Branger, C. Kharif, and N. Akhmediev

Citation: Physics of Fluids (1994-present) 25, 101704 (2013); doi: 10.1063/1.4824706

View online: http://dx.doi.org/10.1063/1.4824706

View Table of Contents: http://scitation.aip.org/content/aip/journal/pof2/25/10?ver=pdfcov

Published by the AIP Publishing

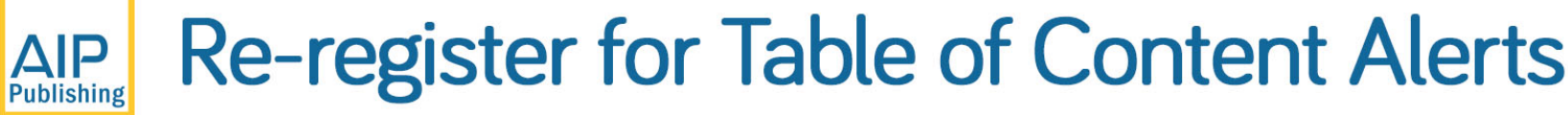

\section{Create a profile.}




\title{
Experiments on wind-perturbed rogue wave hydrodynamics using the Peregrine breather model
}

\author{
A. Chabchoub, ${ }^{1,2, a)}$ N. Hoffmann, ${ }^{2,3}$ H. Branger, ${ }^{4}$ C. Kharif, ${ }^{4}$ \\ and N. Akhmediev 5 \\ ${ }^{1}$ Centre for Ocean Engineering Science and Technology, Swinburne University \\ of Technology, Hawthorn, Victoria 3122, Australia \\ ${ }^{2}$ Department of Mechanical Engineering, Imperial College London, \\ London SW7 2AZ, United Kingdom \\ ${ }^{3}$ Dynamics Group, Hamburg University of Technology, 21073 Hamburg, Germany \\ ${ }^{4}$ Aix Marseille Université, CNRS, Centrale Marseille, IRPHE UMR 7342, \\ 13384, Marseille, France \\ ${ }^{5}$ Optical Sciences Group, Research School of Physics and Engineering, Institute of Advanced \\ Studies, The Australian National University, Canberra ACT 0200, Australia
}

(Received 28 June 2013; accepted 12 September 2013; published online 10 October 2013)

\begin{abstract}
Being considered as a prototype for description of oceanic rogue waves, the Peregrine breather solution of the nonlinear Schrödinger equation has been recently observed and intensely investigated experimentally in particular within the context of water waves. Here, we report the experimental results showing the evolution of the Peregrine solution in the presence of wind forcing in the direction of wave propagation. The results show the persistence of the breather evolution dynamics even in the presence of strong wind and chaotic wave field generated by it. Furthermore, we have shown that characteristic spectrum of the Peregrine breather persists even at the highest values of the generated wind velocities thus making it a viable characteristic for prediction of rogue waves. @ 2013 AIP Publishing LLC. [http://dx.doi.org/10.1063/1.4824706]
\end{abstract}

Ocean rogue waves (RWs) ${ }^{1}$ are known to appear suddenly from nowhere and to disappear without a trace. Within the framework of exact breather solutions of the nonlinear Schrödinger equation (NLS) on finite background, ${ }^{2-4} \mathrm{RWs}$ have indeed this typical feature. Recent observations of doubly-localized solutions of the $\mathrm{NLS}^{5-8}$ confirmed the fact that the long period limit of modulation instability (MI), also referred to as the Benjamin-Feir instability, ${ }^{9}$ may be the key object to discuss within the framework of the NLS, a weakly nonlinear evolution equation describing the dynamics of wave packets in nonlinear dispersive media. Breather solutions are therefore under intensive study due to analogies that can be established for several nonlinear dispersive media. ${ }^{4,10,11}$ Being localized in time and in space, the Peregrine breather solution of $\mathrm{NLS}^{3}$ is considered to be an appropriate model to describe the dynamics of RWs since in addition to its double localization, it amplifies the carrier by a factor of three. Finally, wave flume experiments ${ }^{6}$ proved its physical validity in water waves and it is nowadays used as a model to analyse the impact of RWs on ships. ${ }^{12}$ Here, we present experimental results showing the evolution of the Peregrine breather in the presence of weak as well as strong wind forcing. The results show the robustness of the Peregrine solution with respect to either of these perturbations. Furthermore, it is shown that the shape of the main frequency peak in the frequency spectrum is invariant to the level of forcing. This work may motivate further analytical and numerical work in order to confirm these experimental findings.

The effect of wind blowing over packets of surface water waves is twofold: (i) it produces a variation in the pressure exerted on the surface that results in a flux of energy from the wind to the waves and (ii) it generates a rotational current in the water. Generally, the MI is considered independently of the effects of wind. The question is: How the presence of wind may modify this kind of instability? Note that in addition to these two effects, the wind produces randomness

\footnotetext{
a)Electronic mail: achabchoub@swin.edu.au
} 
at the sea surface, too. It was shown that the effect of randomness characterized by the spectral bandwidth is to reduce the growth rate and extent of the instability compared to the regular case, using a simplified nonlinear spectral transport equation. ${ }^{13}$ These results were confirmed later using the Zakharov equation. ${ }^{14}$ Later on, a series of experiments on mechanically generated waves with wind blowing over them ${ }^{15,16}$ confirmed that gentle wind tends to enhance sideband instability, whereas stronger wind tends to reduce or suppress MI. ${ }^{17-19}$ An experimental investigation on the initial instability of nonlinear deep-water wave trains ${ }^{20}$ was not in agreement with the previous results since it was shown that wind did not inhibit the growth of the sideband. Nevertheless, for a quite strong wind a disappearance of the sideband was observed and the fact that the wind may reduce the naturally developed initial sideband energy, while the growth rate is not necessarily suppressed, was reported. The latter authors emphasized that further study of the natural selection process of the sideband in the tank is crucial for estimating what may happen in the ocean. Numerical simulations on the interaction of wind and extreme gravity waves due to MI were reported in Ref. 21, using the modified Jeffreys' sheltering mechanism as wind modelling. Contrary to the case without wind, it was observed that the wind sustains the maximum of modulation due to the MI. Another experimental and numerical investigation on the influence of wind on extreme wave events due to dispersive focusing and MI as well, by use of the modified Jeffreys' sheltering theory as windmodel, demonstrated that wind blowing over modulated wave trains may increase the time duration and the amplitude of extreme wave events. ${ }^{22}$ The theoretical analysis on the MI in deep-water under the action of wind and dissipation by deriving a perturbed $\mathrm{NLS}^{23}$ showed that the MI of the wave group depends on both: the frequency of the carrier wave and the strength of the friction velocity. Using a fully nonlinear approach the theoretical work has been developed to the case of strongly nonlinear waves. ${ }^{24,25} \mathrm{~A}$ few papers have been devoted to describe the influence of the mentioned vorticity in water on the MI, for instance. ${ }^{26}$ Although the condition of linear stability of the nonlinear plane wave solution is provided in a complicated way, the latter author did not develop a detailed stability analysis as a function of the vorticity and depth. Considering the instability properties of weakly nonlinear wave packets to three-dimensional disturbances in the presence of shear, the system of equations reduces to the familiar NLS equation, when confining the evolution to be purely two-dimensional, and a stability analysis for the case of a linear shear was as well illustrated in Ref. 27. Within the framework of deep-water, the MI of a Stokes wave train in uniform velocity shear has been studied. ${ }^{16}$ The coefficient of the nonlinear term of the NLS equation the authors derived was erroneous, as noted in Ref. 28. The latter author investigated the effect of piecewise-linear velocity profiles in water of infinite depth on sideband instability of a finite-amplitude gravity wave, while the coefficients of the derived NLS equation were computed numerically for specific values of the vorticity and depth of shear layer. In Ref. 29, the author considered the MI of a modulated wave train in both positive and negative shear currents within the framework of the fully nonlinear water wave equations. For a given wave steepness, the results have been compared with the irrotational case and it has been shown that the envelope of the modulated wave train grows faster in a positive shear current and slower in a negative shear current. Using the fully nonlinear equations, a numerical investigation of some instability characteristics of two-dimensional finite amplitude surface waves on a linear shearing flow to three-dimensional infinitesimal rotational disturbances was reported in Ref. 30. Very recently, a NLS equation in the presence of a vertically sheared current of constant vorticity was derived. ${ }^{31}$ The results of the study prove that the presence of vorticity modifies significantly the MI properties of weakly nonlinear plane waves, namely, the growth rate and bandwidth. As we can see from the above references, there is a solid body of research on the influence of wind on MI. Some results contradict the others and further work is needed to clarify the issue. However, here, we do not intend to do this. Instead, we concentrate just on the limiting case of MI when its period goes to infinity. Our aim is to study, experimentally, the influence of wind on the evolution of the Peregrine breather once it was initiated with the small amplitude perturbation.

The MI can be described within the framework of deep-water waves which are described by the $\mathrm{NLS}^{32}$ given by

$$
i\left(\frac{\partial \psi}{\partial t}+c_{g} \frac{\partial \psi}{\partial x}\right)-\frac{\omega_{0}}{8 k_{0}^{2}} \frac{\partial^{2} \psi}{\partial x^{2}}-\frac{\omega_{0} k_{0}^{2}}{2}|\psi|^{2} \psi=0
$$




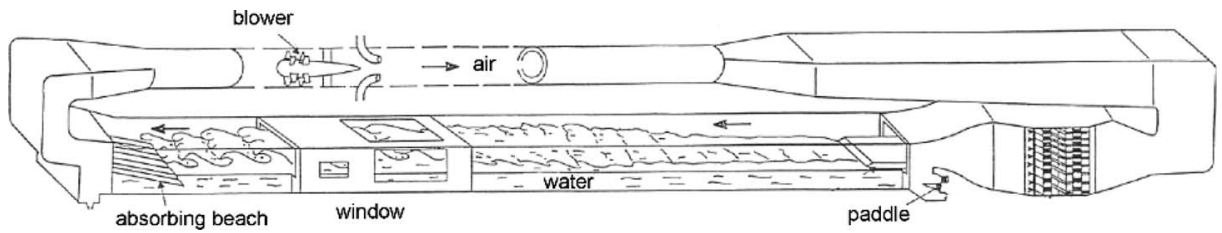

FIG. 1. Schematic illustration of the wind wave facility. ${ }^{4}$

where $\psi$ denotes the wave envelope, while $\omega_{0}$ and $k_{0}$ are the wave frequency and wave number, respectively. They are linked through the dispersion relation: $\omega_{0}=\sqrt{g k_{0}}$, where $g$ is the gravitational acceleration. It is well known that the plane wave solution $\psi(x, t)=a_{0} \exp \left(-i \frac{k_{0}^{2} a_{0}^{2} \omega_{0} t}{2}\right)$ of the NLS with arbitrary amplitude $a_{0}$ is unstable relative to periodic modulations within certain range of wavenumbers. ${ }^{10}$ The MI can be also discussed in terms of breather solutions on finite background..$^{2,3}$ First-order family of breather solutions, describing the exact Benjamin-Feir mechanism, are known as Akhmediev breathers. ${ }^{2}$ These solutions are periodic in space and have specific growth-decay evolution in time. For each specific Akhmediev breather, i.e., for each specific modulation period, the maximal amplitude amplification of the carrier is bounded by one and three. ${ }^{2,11}$ The limiting case of the Peregrine breather solution ${ }^{3}$ corresponds to the case when the modulation period becomes infinite and is given in dimensional units by

$$
\psi_{P}(x, t)=a_{0} \exp \left(-i \frac{k_{0}^{2} a_{0}^{2} \omega_{0}}{2} t\right)\left(-1+\frac{4\left(1-i k_{0}^{2} a_{0}^{2} \omega_{0} t\right)}{1+\left[2 \sqrt{2} k_{0}^{2} a_{0}\left(x-c_{g} t\right)\right]^{2}+k_{0}^{4} a_{0}^{4} \omega_{0}^{2} t^{2}}\right) .
$$

This doubly-localized breather solution ${ }^{6}$ amplifies the carrier by an exact factor of three. As such, it is considered to be an appropriate prototype to describe ocean rogue waves. As we show in the present work, the Peregrine breather once initiated is a robust formation and can survive even in a chaotic wave field generated by the wind.

Experiments have been conducted in a closed wind wave facility installed at IRPHE/Luminy Marseille. A schematic illustration is shown in Fig. $1 .{ }^{4}$ The tank is $40 \mathrm{~m}$ long and $3 \mathrm{~m}$ wide. The water is $0.9 \mathrm{~m}$ deep, and the air channel is $1.5 \mathrm{~m}$ high. The tank is equipped with a controlled recirculating wind tunnel which can generate wind speeds between 1 and $14 \mathrm{~ms}^{-1}$. A computer-controlled wavemaker can generate waves in a frequency range from $0.6 \mathrm{~Hz}$ to $2.5 \mathrm{~Hz}$. It is entirely submerged under the upstream beach to avoid any perturbation of the air flow which could be induced by its displacement. The upwind end of the tank is specially profiled to ensure minimum disturbance to either the generation of mechanical waves or the turbulent boundary layer in the airflow above the waves. The tunnel roof is carefully profiled to create an airflow boundary layer of zero pressure gradient along the test section. At the downwind end of the tank a permeable absorbing beach was installed to minimize wave reflection. A complete description of the tank can be found in Ref. 33 . Wind speed was measured with a Pitot tube. We used one capacitance wave gauge and six resistive wave gauges to measure the water elevation at different locations inside the tank in order to see in the advanced stages of wave evolution in more detail, see Table I. The accuracy of the water elevation measurement is about $0.3 \mathrm{~mm}$. The boundary condition, applied to the flap, is determined as the surface elevation, given in the first-order in steepness at a specific position $x^{*}$,

$$
\eta\left(x^{*}, t\right)=\operatorname{Re}\left(\psi_{P}\left(x^{*}, t\right) \cdot \exp \left[i\left(k_{0} x^{*}-\omega_{0} t\right)\right]\right) .
$$

TABLE I. Positions (P) of the gauge number (GN) along the tank.

\begin{tabular}{cccccccc}
\hline \hline GN & 1 & 2 & 3 & 4 & 5 & 6 & 7 \\
\hline $\mathrm{P}(\mathrm{m})$ & 2.06 & 8.09 & 8.81 & 9.50 & 10.41 & 11.08 & 11.71 \\
\hline \hline
\end{tabular}




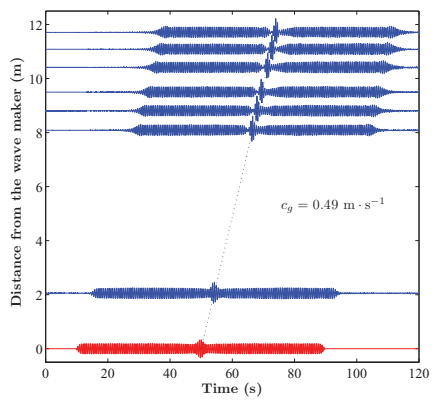

FIG. 2. Evolution of the Peregrine breather for the carrier parameters $a_{0}=0.75 \mathrm{~cm}$ and $\varepsilon:=a_{0} k_{0}=0.08$ without wind action, measured starting $2.06 \mathrm{~m}$ from the flap (darker gray line/blue), while the dynamics is initiated from a small amplitude amplification as initial conditions applied to the flap (lighter gray line/red). The group velocity measured experimentally is $c_{g}=0.49 \mathrm{~ms}^{-1}$.

All experiments have been conducted for a carrier amplitude of $a_{0}=0.75 \mathrm{~cm}$, while the wave frequency and wave number were chosen to be $\omega_{0}=10.68 \mathrm{rad} \cdot \mathrm{s}^{-1}$ and $k_{0}=11.63 \mathrm{rad} \cdot \mathrm{m}^{-1}$, respectively.

The first set of experiments was conducted in order to reproduce the Peregrine breather the same way as reported in Ref. 6 but in the water tank described above. Namely, first, we excited the Peregrine breather without wind forcing. With the initial condition corresponding to the Peregrine breather at the wave generator position, the maximal amplitude amplification of three is expected at a position of $10.4 \mathrm{~m}$ from the paddle. Fig. 2 shows this wave train evolution along the flume. Indeed, we observed the Peregrine breather dynamics and the measurements at the gauge 5 confirmed that the maximal amplification of three is reached. The group velocity is calculated from the Hilbert transform of the cross-correlation function of the measured water levels at two different locations. In the absence of wind, we measured the group velocity to be $c_{g}=0.49 \mathrm{~ms}^{-1}$, which nearly coincides with the theoretical group velocity given by the dispersion relation to second-order in steepness. Here, we concentrate our attention on the advanced stages of the evolution of the breather measured by the gauges $2,3,4,5,6$, and 7 , installed at the positions between 8 and $12 \mathrm{~m}$ from the wave generator. In the following experiments, we increased, step by step, the constant wind speed $U$ blowing in the direction of wave propagation for the same initial conditions. The wind naturally distorts the background wave train as we can see from Fig. 3. The higher the wind speed, the higher are these distortions, which are generally chaotic. Nevertheless, the initial small modulation grows and its highest amplitude prevails in the chaotic wave field of the background. Furthermore, Fig. 3 shows that even in the presence of the strongest wind with the speed of $9 \mathrm{~ms}^{-1}$, the Peregrine breather dynamics engenders the formation of a large amplitude wave, which stands out against the chaotic background wave train. In order to confirm this fact, which perhaps cannot be clearly appreciated from the relatively low resolution traces in Fig. 3(d), we show separately the trace measured by the gauge 3 from the above set in Fig. 4. We can see clearly, that the Peregrine breather appears as a large amplitude wave, which is significantly higher than the rest of the surrounding waves. The maximal breather amplitude changes, of course, when the wind is present. The maximal amplitude of the waves as well as the standard deviation of the surface elevation for the corresponding wind speed are shown in Table II. Remarkably, they are higher than in the absence of the wind, which is providing an additional amplification of the breather. Here, it has to be mentioned that these amplifications may be much more significant since recurrent breaking is observed during the experiments ${ }^{34}$ and it cannot be confirmed that the wave gauges, fixed at a specific position, measured the breather's amplitude at its maximal local steepness, i.e., just before breaking. These observations are in line with the analytical results reported in Refs. 21,23, and 35, which predicted that the wind accelerates the evolution of the MI. Furthermore, the presented experimental results confirm the work of Ref. 20 and validate the fact that the effect of even strong winds on the MI is not dominant and indeed small. Another visible effect caused by the wind is the increase of the group velocity. Measured group velocities are: $c_{g}=0.49 \mathrm{~ms}^{-1}$ for $U=0 \mathrm{~ms}^{-1} ; c_{g}=0.53 \mathrm{~ms}^{-1}$ for $U=3 \mathrm{~ms}^{-1}$; 

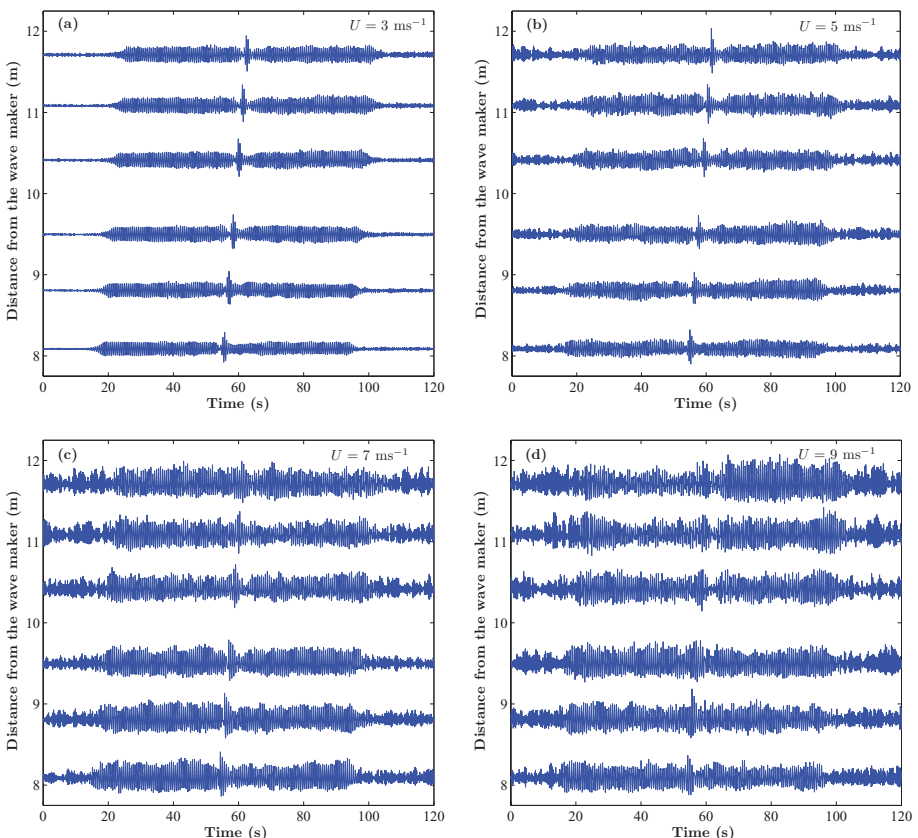

FIG. 3. Advanced stages of the evolution of the Peregrine breather for (a) a wind speed value of $U=3 \mathrm{~ms}^{-1}$, (b) $U=5 \mathrm{~ms}^{-1}$, (c) $U=7 \mathrm{~ms}^{-1}$, and (d) $U=9 \mathrm{~ms}^{-1}$. These observations show that despite the presence of a strong wind and related chaotization of the background, the theoretical Peregrine breather dynamics still reasonably well defines the formation of the centrally located RW.

$c_{g}=0.54 \mathrm{~ms}^{-1}$ for $U=5 \mathrm{~ms}^{-1} ; c_{g}=0.56 \mathrm{~ms}^{-1}$ for $U=7 \mathrm{~ms}^{-1} ; c_{g}=0.59 \mathrm{~ms}^{-1}$ for $U=9 \mathrm{~ms}^{-1}$. As explained by Ref. 21, the increase of velocity is due to the wind-induced surface-drift current which is proportional to the wind drag velocity. Here, we should also mention that increasing the initial steepness of the background wave may cause an early breaking of the waves and the Peregrine-type RWs would be difficult to observe. ${ }^{36,37}$ Another unique feature of the Peregrine breather potentially important for its prediction is its characteristic triangular spectrum co-located on top with the main frequency component of the background wave. ${ }^{38}$ The latter is a delta-function in frequency domain in case of an infinite monochromatic background. If we ignore or eliminate the central peak caused by the background, the Peregrine breather dynamics can be easily identified from the experimental triangular spectra. ${ }^{39}$ Our present experiments show these spectra which are also slightly distorted by the wind. Fig. 5 shows the power spectra of several wave train records, measured with the first gauge. In the absence of the wind, i.e., for $U=0 \mathrm{~ms}^{-1}$, we can clearly notice the main triangular spectrum at $1.7 \mathrm{~Hz}$. The total energy of the wave trains increases with the speed of the wind and the corresponding spectral curves show higher powers. For nonzero wind speeds up to $9 \mathrm{~ms}^{-1}$ the main

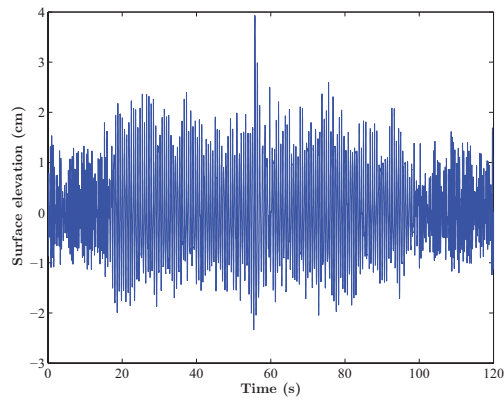

FIG. 4. The measurements shown in Fig. 3(d) recorded by the gauge 3 with higher resolution along the vertical axis. The largest wave appears at this position at $t=55 \mathrm{~s}$. 
TABLE II. Maximal measured wave amplitudes and standard deviation of the wave train versus corresponding wind speed.

\begin{tabular}{llllll}
\hline \hline Wind speed in $\mathrm{ms}^{-1}$ & 0 & 3 & 5 & 7 & 9 \\
\hline Maximal wave amplitude in cm & 2.3 & 2.7 & 3.3 & 3.4 & 3.9 \\
Standard deviation of the surface elevation & 0.37 & 0.44 & 0.53 & 0.77 & 0.80 \\
\hline \hline
\end{tabular}

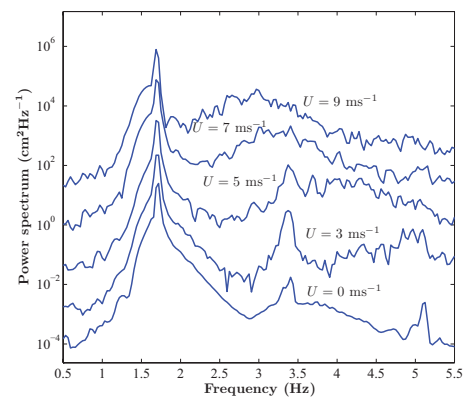

FIG. 5. Power spectra of the first gauge record for the different wind speed values.

triangular feature of spectrum at $1.7 \mathrm{~Hz}$ does not disappear. When increasing the wind speed, the total energy of the wave train simply grows but the triangular shapes on top of the main frequency are preserved. This demonstrates that the Peregrine breather is present in the wave train no matter if the wind is blowing or not. These experiments justify once again the promising idea of using the spectral measurements of the doubly-localized breather solutions for a possible early prediction of rogue waves even in presence of winds and a chaotic background makes their measurements possible viable candidates for the rogue wave predictions. ${ }^{40}$ Actual realizations of wave trains with a given spectrum may vary due to various phase relations between the spectral components, and the triangular spectra do not always correspond to Peregrine breather dynamics. In case of chaotic wave fields used in simulations of Ref. 40, phase matching of various spectral components may start by chance and can be amplified due to the nonlinearity. Clearly, more detailed studies continuing those in Ref. 40 would be needed in order to use the spectral measurements in practice.

To summarize, we report an experimental investigation of the Peregrine breather evolution in a wave tank in presence of wind. Our study shows that slow winds in propagation direction do not have significant influence on the evolution of the breather. Furthermore, under the action of strong winds, a formation of a Peregrine breather-like large wave amplitude can be clearly observed. The spectral measurements at the beginning of the breather evolution show a typical triangular spectra co-located with the main frequency component of the background wave. This spectral feature does not disappear when the wind speed is increased. Thus, the MI dynamics of the Peregrine breather dynamics remains valid.

A.C. would like to thank Efim Pelinovsky for helpful discussions. N.H. and N.A. acknowledge the support of the Volkswagen Stiftung. N.A. acknowledges partial support of the Australian Research Council (Discovery Project No. DP110102068). A.C., C.K., and N.A. acknowledge support from the Fields Institute. N.A. acknowledges support from the Alexander von Humboldt Foundation.

${ }^{1}$ C. Kharif and E. Pelinovsky, "Physical mechanisms of the rogue wave phenomenon," Eur. J. Mech. B/Fluids 22, 603-634 (2003).

${ }^{2}$ N. Akhmediev, V. M. Eleonskii, and N. E. Kulagin, "Exact first-order solutions of the nonlinear Schrödinger equation," Teor. Matem. Fiz. (USSR) 72, 183-196 (1987) Theor. Math. Phys. 72, 809-818 (1988) (in English).

${ }^{3}$ D. H. Peregrine, "Water waves, nonlinear Schrödinger equations and their solutions," J. Austral. Math. Soc. Ser. B 25, $16-43$ (1983).

${ }^{4}$ C. Kharif, E. Pelinovsky, and A. Slunyaev, Rogue Waves in the Ocean (Springer, Heidelberg, NY, 2009).

${ }^{5}$ B. Kibler, J. Fatome, C. Finot, G. Millot, F. Dias, G. Genty, N. Akhmediev, and J. M. Dudley, "The Peregrine soliton in nonlinear fibre optics," Nat. Phys. 6, 790-795 (2010). 
${ }^{6}$ A. Chabchoub, N. P. Hoffmann, and N. Akhmediev, "Rogue wave observation in a water wave tank," Phys. Rev. Lett. 106, $204502(2011)$

${ }^{7}$ H. Bailung, S. K. Sharma, and Y. Nakamura, "Observation of Peregrine solitons in a multicomponent plasma with negative ions," Phys. Rev. Lett. 107, 255005 (2011).

${ }^{8}$ A. Chabchoub, N. Hoffmann, M. Onorato, and N. Akhmediev, "Super rogue waves: Observation of a higher-order breather in water waves," Phys. Rev. X 2, 011015 (2012).

${ }^{9}$ T. B. Benjamin and J. E. Feir, "The disintegration of wave trains on deep water. Part 1," Theory. J. Fluid Mech. 27, 417-430 (1967).

${ }^{10}$ A. Osborne, Nonlinear Ocean Waves and the Inverse Scattering Transform (Elsevier, Amsterdam, 2010).

${ }^{11}$ M. Onorato, S. Residori, U. Bortolozzo, A. Montina, and F. T. Arecchie, "Rogue waves and their generating mechanisms in different physical contexts," Phys. Rep. 528, 47-89 (2013).

${ }^{12}$ M. Onorato, D. Proment, G. Clauss, and M. Klein, "Rogue waves: From nonlinear Schrödinger breather solutions to sea-keeping test," PLoS ONE 8(2), e54629 (2013).

${ }^{13}$ I. E. Alber, "The effects of randomness on the stability of two-dimensional surface wavetrains," Proc. R. Soc. London A 363, 525-546 (1978).

${ }^{14}$ D. R. Crawford, P. G. Saffman, and H. C. Yuen, "Evolution of random inhomogeneous field of nonlinear deep-water gravity waves," Wave Motion 2, 1-16 (1980).

${ }^{15}$ L. F. Bliven, N. E. Huang, and S. R. Long, "Experimental study of the influence of wind on Benjamin-Feir sideband instability," J. Fluid Mech. 162, 237-260 (1986)

${ }^{16}$ J. C. Li, W. H. Hui, and M. A. Donelan, "Effects of velocity shear on the stability of surface deep water wave trains," in Nonlinear Water Waves, edited by K. Horikawa and H. Maruo (Springer, Berlin, 1987), pp. 213-220.

${ }^{17}$ T. Hara and C. C. Mei, "Frequency downshift in narrowbanded surface waves under the influence of wind," J. Fluid Mech. 230, 429-477 (1991).

${ }^{18}$ K. Trulsen and K. B. Dysthe, "Action of windstress and breaking on the evolution of a wavetrain," in Breaking Waves, edited by M. L. Banner and R. H. J. Grimshaw (Springer-Verlag, Berlin, 1992), pp. 243-249.

${ }^{19}$ A. Galchenko, A. V. Babanin, D. Chalikov, I. R. Young, and B. K. Haus, "Influence of wind forcing on modulation and breaking of one-dimensional deep-water wave groups,” J. Phys. Oceanogr. 42, 928-939 (2012).

${ }^{20}$ T. Waseda and M. P. Tulin, "Experimental study of the stability of deep-water wave trains including wind effects," J. Fluid Mech. 401, 55-84 (1999).

${ }^{21}$ J. Touboul and C. Kharif, "On the interaction of wind and extreme gravity waves due to modulational instability," Phys. Fluids 18, 108103 (2006).

${ }^{22}$ C. Kharif, J. P. Giovanangel, J. Touboul, L. Grare, and E. Pelinovsky, "Influence of wind on extreme wave events: Experimental and numerical approaches,” J. Fluid Mech. 594, 209-247 (2008).

${ }^{23}$ C. Kharif, R. Kraenkel, M. Manna, and R. Thomas, "Modulational instability in deep water under the action of wind and dissipation,” J. Fluid Mech. 664, 138-149 (2010).

${ }^{24}$ C. Kharif and J. Touboul, "Under which conditions the Benjamin-Feir instability may spawn an extreme wave event: A fully nonlinear approach,” Eur. Phys. J. Spec. Top. 185, 159-168 (2010).

${ }^{25}$ J. Touboul and C. Kharif, "Nonlinear evolution of the modulational instability under weak forcing and dissipation," Nat. Hazards Earth Syst. Sci. 10, 2589-2597 (2010).

${ }^{26}$ R. S. Johnson, "On the modulation of water waves on shear flows," Proc. R. Soc. London A 347, 537-546 (1976).

${ }^{27}$ M. Oikawa, K. Chow, and D. J. Benney, "The propagation of nonlinear wave packets in a shear flow with a free surface," Stud. Appl. Math. 76, 69-92 (1987).

${ }^{28}$ A. I. Baumstein, "Modulation of gravity waves with shear in water," Stud. Appl. Math. 100, 365-390 (1998)

${ }^{29}$ W. Choi, "Nonlinear surface waves interacting with a linear shear current," Math. Comput. Simul. 80, 101-110 (2009).

${ }^{30}$ M. Okamura and M. Oikawa, "The linear stability of finite amplitude surface waves on a linear shearing flow," J. Phys. Soc. Jpn. 58, 2386-2396 (1989).

${ }^{31}$ R. Thomas, C. Kharif, and M. Manna, "A nonlinear Schrödinger equation for water waves on finite depth with constant vorticity," Phys. Fluids 24, 127102 (2012).

${ }^{32}$ V. E. Zakharov, "Stability of periodic waves of finite amplitude on a surface of deep fluid," J. Appl. Mech. Tech. Phys. 9, 190-194 (1968).

${ }^{33}$ M. Coantic, A. Ramamonijiarisoa, P. Mestayer, F. Resch, and A. Favre, "Wind-water tunnel simulation of small-scale ocean-atmosphere interactions," J. Geo. Phys. 86, 6607-6626 (1981).

${ }^{34}$ A. Iafrati, A. Babanin, and M. Onorato, "Modulational instability, wave breaking, and formation of large-scale dipoles in the atmosphere," Phys. Rev. Lett. 110, 184504 (2013).

${ }^{35} \mathrm{M}$. Onorato and D. Porment, "Approximate rogue wave solutions of the forced and damped nonlinear Schrödinger equation for water waves," Phys. Lett. A 376(45), 3057-3059 (2012).

${ }^{36}$ M. A. Donelan, A. V. Babanin, I. R. Young, and M. L. Banner, "Wave-follower field measurements of the wind-input spectral function. Part II: Parameterization of the wind input," J. Phys. Oceanogr. 36, 1672-1689 (2006).

${ }^{37}$ A. V. Babanin, D. Chalikov, I. R. Young, and I. Savelyev, "Predicting the breaking onset of surface water waves," Geophys. Res. Lett. 34, L07605, doi:10.1029/2006GL029135 (2007).

${ }^{38}$ N. Akhmediev, A. Ankiewicz, J. M. Soto-Crespo, and J. M. Dudley, "Rogue wave early warning through spectral measurements?," Phys. Lett. A 375, 541-544 (2011).

${ }^{39}$ A. Chabchoub, N. P. Hoffmann, and N. Akhmediev, "Spectral properties of the Peregrine soliton observed in a water wave tank," J. Geophys. Res. 117, C00J03, doi:10.1029/2011JC007671 (2012).

${ }^{40}$ N. Akhmediev, J. M. Soto-Crespo, A. Ankiewicz, and N. Devine, "Early detection of rogue waves in a chaotic wave field," Phys. Lett. A 375, 2999-3001 (2011). 